\title{
FEEDBACK LINEARIZATION AND BACKSTEPPING CONTROLLERS FOR COUPLED TANKS
}

\author{
Bachir Nail $^{1,3}$, Belkacem Bekhiti ${ }^{2}$, Ahmed Hafaifa ${ }^{1,3}$, Abdellah Kouzou ${ }^{1,3}$ and \\ Nadji Hadroug ${ }^{1,3}$ \\ ${ }^{1}$ Science and Technology Department, University of ZianeAchour, Moudjbara Street BP \\ 3117 Djelfa, Algeria . \\ ${ }^{2}$ Electronics and Electrotechnics Institute, University of Boumerdes, Boumerdes 35000, \\ Algeria. \\ ${ }^{3}$ The Applied Automation and Industrial Diagnostics Laboratory LAADI, University of \\ DJELFA, Algeria.
}

\begin{abstract}
This paper investigates the usage of some sophisticated and advanced nonlinear control algorithms inorder to control a nonlinear Coupled Tanks System. The first control procedure is called the Feedbacklinearisation control (FLC), this type of control has been found a successful in achieving a global exponentialasymptotic stability, with very short time response, no significant overshooting is recordedand with a negligible norm of the error. The second control procedure is the approaches of Backsteppingcontrol $(B C)$ which is a recursive procedure that interlaces the choice of a Lyapunov functionwith the design of feedback control, from simulation results it shown that this method preserves tracking, robust control and it can often solve stabilization problems with less restrictive conditions may beencountered in other methods. Finally both of the proposed control schemes guarantee the asymptoticstability of the closed loop system meeting trajectory tracking objectives.
\end{abstract}

\section{KEYWORDS}

Feedback linearization, Backstepping, Lyapunov candidate function, Coupled tanks system, Nonlinearcontrol.

\section{INTRODUCTION}

The modeling and the control of industrial process, present a big challenge, hence the reservoirs andhydraulic systems are considered an important part in them, so the selection of the coupled tanks isnot accidental for several reasons, first the coupled tanks are very useful in industry, in the researchlaboratories in petroleum reservoir engineering and in nuclear reactor, the second reason is related bythe behavior of this systems in general, we see the dynamic model of the coupled tanks is nonlinearand contains a square root in mathematical differential equations that considered a good example fortesting the stability and the robustness of any control.

There are several works and researches and scientific articles have preceded us to work on this systemand they are applied too many controllers (linear and nonlinear). Among these works, 
Sliding ModeControl And Optimal GPC Algorithm for Coupled Tanks [1], Modelling and Control of Coupled Tank Liquid Level System using Backstepping Method [2], Model and Control for Coupled Tanks using Labview [3], A Neuro-fuzzy sliding Mode Controller Using Nonlinear Sliding Surface Applied to theCoupled Tanks System [4], and Nonlinear constrained predictive control [5].In this paper we haveused efficient feedback linearization control without transformation, and the we have ameliorate the quality of the signal control and the statical error in the two controllers, compared to the previous works [1-5].

The paper is organized as follows. The first section contains the system model, in the form of nonlineardifferential equations, and the structure of coupled tanks. The second section, gives somepreliminary of feedback linearization approach [6],[7], and how do we apply these control on this system, explaining that in scheme block diagram, and simulation results with some comments on them. The third section, We dealt also about the backstepping approach [8],[9] and its theory in second order system, then the application of this control on coupled tanks , explaining that in scheme block diagram, and simulation results with some comments on them. Finally, the fourth section is a conclusion, containing a comparison between the two approaches, and highlights the pros and cons of each in their application the coupled tanks.

\section{WATER TANK MODEL}

The schematic drawing in (Figure.1) represents the model of a two degree of freedom (DOF) statecoupled,water tank system [10]:

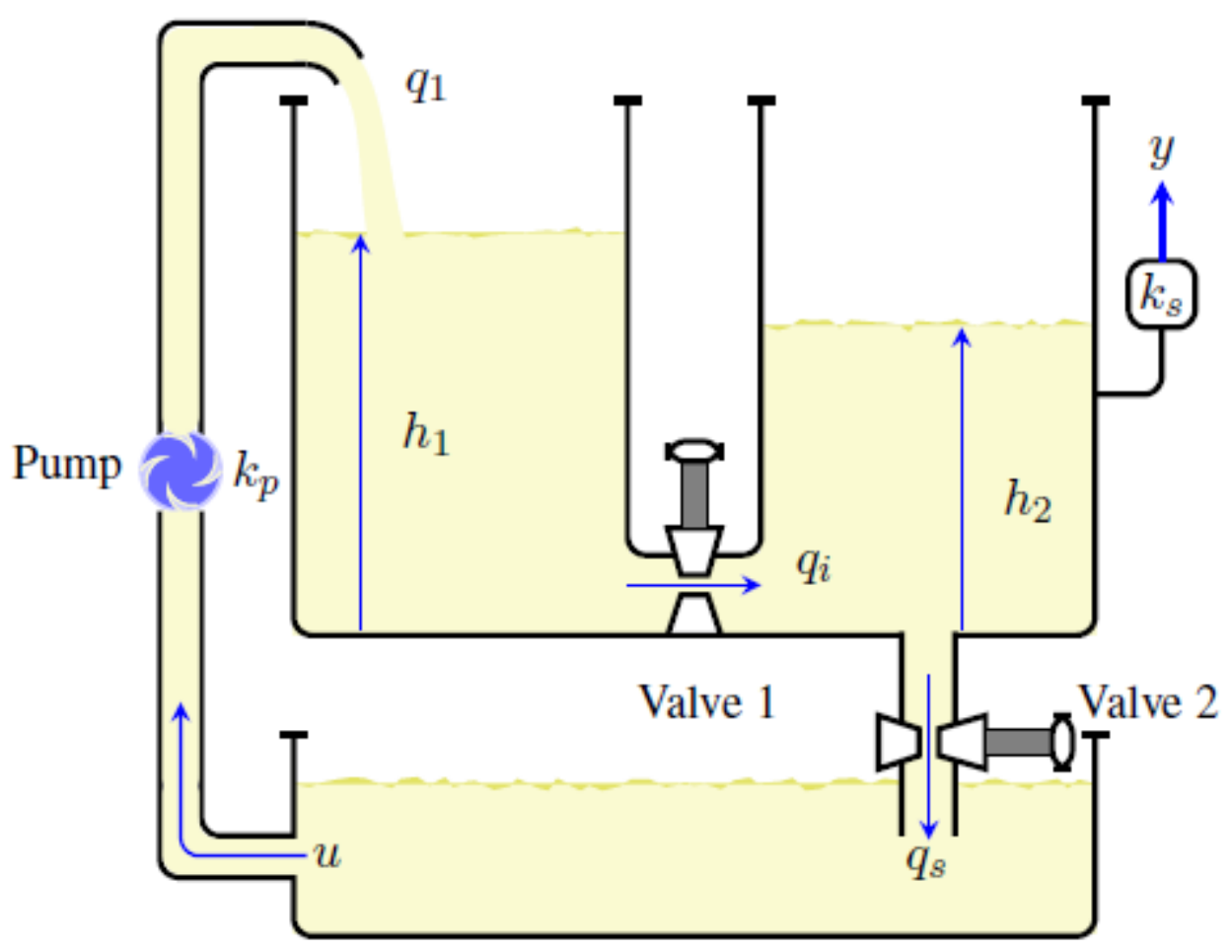

Figure 1: Coupled Tanks System. 
The coupled tank system is defined by the following nonlinear differential equations:

$$
\begin{aligned}
\frac{d h_{1}}{d t} & =\frac{1}{c}\left(-s_{1} a_{i} \sqrt{2 g\left(h_{1}-h_{2}\right)}+k_{p} u\right) \\
\frac{d h_{2}}{d t} & =\frac{1}{c}\left(s_{1} a_{i} \sqrt{2 g\left(h_{1}-h_{2}\right)}-s_{2} a_{s} \sqrt{2 g h_{2}}\right) \\
y & =k_{s} h_{2}
\end{aligned}
$$

With

$$
u=\left\{\begin{array}{lll}
u_{\max } & \text { if } & u \geq u_{\max } \\
0 & \text { if } & u \leq 0
\end{array}\right.
$$

where $h_{1,2}$ are liquid levels in the tanks 1,2 respectively, $\mathrm{g}$ is the gravitational constant, $s_{1,2}$ channel sectionalarea, $k_{p}, k_{s}$ arepumpandsensorgains, and $q_{i}$ representtheflowrate.

By Letting $x_{1}=h_{2}, x_{2}=h_{1}$, and the output $y=k_{s} h_{2}$, the dynamic representation of our system will beinthenonlinearstatespaceformasshown[9]:

$$
\left\{\begin{array}{l}
\dot{x}=f(x)+g(x) u+d \\
y=h(x)
\end{array}\right.
$$

Where $f, g$ and $h$ are sufficiently smooth in a domain $D \subset R n$. The mappings $f: D \rightarrow R n$ and $\mathrm{g}: \mathrm{D} \rightarrow \mathrm{Rn}$ are called vector fields on $\mathrm{D}$.

$$
\begin{gathered}
x=\left[x_{1}, x_{2}\right]^{T}=\left[h_{2}, h_{1}\right]^{T}, \text { such as } \\
\left\{\begin{array}{l}
\dot{x}_{1}=f_{1}(x) \\
\dot{x}_{2}=f_{2}(x)+k_{a} u \\
y=k_{s} x_{1}
\end{array}\right.
\end{gathered}
$$

With

$$
\left\{\begin{array}{l}
f_{1}(x)=\beta_{1} \sqrt{x_{2}-x_{1}}-\beta_{2} \sqrt{x_{1}} \\
f_{2}(x)=-\beta_{1} \sqrt{x_{2}-x_{1}}
\end{array}\right.
$$

And 


$$
\beta_{1}=\frac{s_{1} a_{i} \sqrt{2 g}}{c}, \beta_{2}=\frac{s_{2} a_{s} \sqrt{2 g}}{c}, k_{a}=\frac{k_{p}}{c}
$$

Where

$$
\begin{gathered}
f(x) \triangleq\left[\begin{array}{c}
f_{1}(x) \\
f_{2}(x) \mid
\end{array}\right], g(x) \triangleq\left[\begin{array}{l}
0 \\
k_{a}
\end{array}\right], d \triangleq 0 \\
y=k_{s} x_{1}
\end{gathered}
$$

\section{FEEDBACK LINEARIZATION APPROACH}

Feedback linearization is an approach to nonlinear control design that has attracted lots of research in recent years. The central idea is to algebraically transform nonlinear systems dynamics into (fully or partly) linear ones, so that linear control techniques can be applied.

This differs entirely from conventional (Jacobian) linearization, because feedback linearization is achieved by exact state transformation and feedback, rather than by linear approximations of the dynamics.

\subsection{LIE DERIVATIVES}

Given a scalar function $\mathrm{h}(\mathrm{x})$ and a vector field $\mathrm{f}(\mathrm{x})$, we define a new scalar function $\mathrm{L}_{\mathrm{f}} \mathrm{h}(\mathrm{x})$, called the Lie derivative (or simply, the derivative) of $\mathrm{h}$ with respect to $\mathrm{f}$.

Definition 1: $\mathrm{f}$ is called smooth if $\mathrm{f} \in \mathrm{Rn}$. That $\mathrm{f}$ is continuous and all derivatives of all order are continuous [10].

Definition 2: Let $\mathrm{h}: \mathrm{Rn} \rightarrow \mathrm{R}$ be smooth scalar function, and $\mathrm{f}: \mathrm{Rn} \rightarrow \mathrm{Rn}$ be a smooth vector field on $\mathrm{Rn}$, then the Lie derivative of $\mathrm{h}$ with respect to $\mathrm{f}$ is scalar function defined by [10]:

$$
L_{f} h(x)=(\nabla h) f \text { Where } \nabla h=\partial h / \partial x
$$

Let we choose the level of the second tank $\mathrm{h} 2$ as the controlled output rather than the position control The derivative $y^{*}$ is given by:

$$
\begin{aligned}
\dot{y} & =\frac{\partial h}{\partial x}[f(x)+g(x) u] \\
& =L_{f} h(x)+L_{g} h(x)
\end{aligned}
$$


Where

$$
\frac{\partial h}{\partial x} f(x)=L_{f} h(x) \quad \text { and } \quad \frac{\partial h}{\partial x} g(x)=L_{g} h(x)
$$

$\mathrm{L}_{\mathrm{f}} \mathrm{h}$ : is called the Lie Derivative of $\mathrm{h}$ with respect to $\mathrm{f}$.

If $\operatorname{Lgh}(x)=0$, then $y^{*}=\operatorname{Lf} h(x)$, independent of $u$. If we continue to calculate the second derivative of $y$, denoted by $y(2)$, we obtain:

$$
\begin{aligned}
\dot{y} & =\frac{\partial\left(L_{f} h\right)}{\partial x}[f(x)+g(x) u] \\
& =L_{f}^{2} h(x)+L_{g} L_{f} h(x) u
\end{aligned}
$$

Then $u$ does not appear in the equations of $y, y$ and appears in the equation of $y^{(2)}$ With a nonzero coefficient:

$$
\begin{gathered}
y^{(2)}=L_{f}^{2} h(x)+L_{g} L_{f} h(x) u \\
\ddot{y}=\frac{k_{s} B_{1} k_{a} u}{2 \sqrt{x_{2}-x_{1}}}+\frac{k_{s} B_{1} B_{2} \sqrt{x_{1}}}{2 \sqrt{x_{2}-x_{1}}}-\frac{k_{s} B_{1} B_{2} \sqrt{x_{2}-x_{1}}}{\sqrt{x_{1}}} \\
+\frac{k_{s} B_{2}^{2}}{2}-k_{s} B_{1}^{2}
\end{gathered}
$$

The forgoing equation shows clearly that the system is input-output linearizable, since the state feedback control.

$$
u=\frac{1}{L_{g} L_{f}^{\rho-1} h(x)}\left[-L_{f}^{\rho} h(x)+v(t)\right]
$$

Reduces the input-output map to

$$
y^{(\rho)}=v(t)
$$

Which is a chain of $\rho=2$ integrators. In this case, the integer $\rho$ is called the relative degree of the system.

After some algebraic manipulations and calculations the control input $u$ is given by : 


$$
\frac{1}{\Delta}\left[v(t)+k_{s} B_{1}^{2}-\frac{k_{s} B_{2}^{2}}{2}-\frac{k_{s} B_{1} B_{2} \sqrt{x_{1}}}{2 \sqrt{x_{2}-x_{1}}}+\frac{k_{s} B_{1} B_{2} \sqrt{x_{2}-x_{1}}}{\sqrt{x_{1}}}\right]
$$

Where

$$
\Delta=\frac{k_{s} B_{1} k_{a}}{2 \sqrt{x_{2}-x_{1}}}
$$

and $v$ is an auxiliary control input to be designed. Typically, $v$ is chosenas

$$
v=\ddot{y}_{d}-K_{p}\left(y-y_{d}\right)-K_{v}\left(\dot{y}-\dot{y}_{d}\right)
$$

This yield to the error equation

$$
\ddot{\tilde{y}}+K_{p} \tilde{y}+K_{v} \dot{\tilde{y}}=0
$$

Equation (17) shows that the error dynamics are governed by a linear second order system. Furthermore, if the gains are chosen to be diagonal, then the system will be perfectly decoupled.

By suitable choosing the matrices $\mathrm{Kp}$ and $\mathrm{Kv}$, the tracking error will converge asymptotically to zero. To illustrate this, we can write the error equation (17) in state space representation, i.e. we define the state variable $\mathrm{z}=\left(\mathrm{y}^{\sim}, \mathrm{y}^{\cdots \sim}\right)$, and we can write

$$
\dot{z}=\left[\begin{array}{cc}
0 & I \\
-K_{p} & -K_{v}
\end{array}\right] z=A z
$$

In order to investigate the stability of the equilibrium point, $\mathrm{z}=0$, we choose the Lyapunov function candidate

$$
V(z)=z^{T} P z
$$

Where $P=P^{T}>0$ isthesolutiontoLyapunovequation 


$$
A^{T} P+P A=-Q
$$

With $Q>0$ then, the time derivative of $V(z)$ is

$$
\begin{aligned}
\dot{V}(z) & =\dot{z}^{T} P z+z P \dot{z} \\
& =z^{T} A^{T} P z+z^{T} P A z \\
& =z^{T}\left(A^{T} P+P A\right) z \\
& =-z^{T} Q z
\end{aligned}
$$

Which is negative definite function, from which and according [11] global uniform exponential stability of the equilibrium point follows. (Figure.2) shows the implementation of this feedback linearization

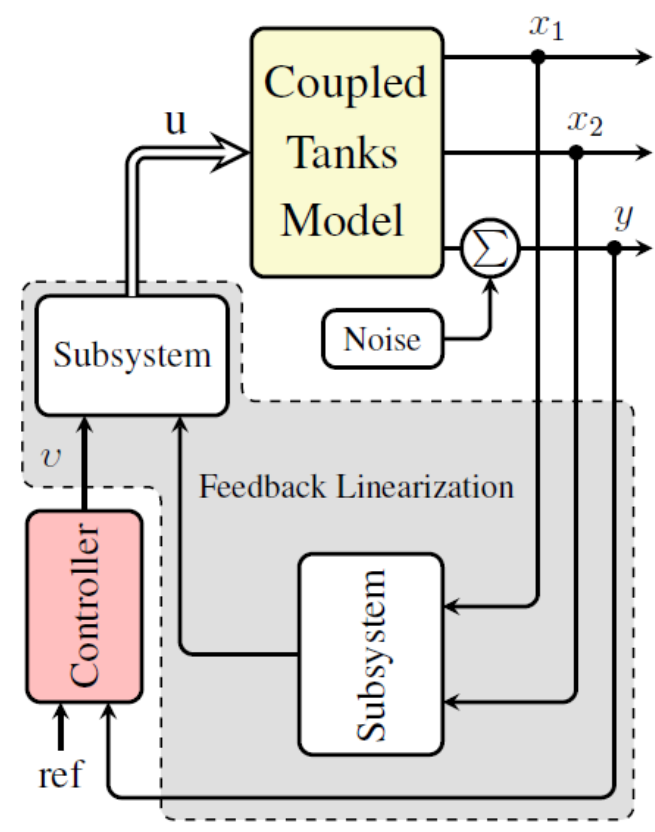

Figure 2: Schematic block diagram of feedback linearization with auxiliary controller

\subsection{SIMULATION RESULTS OF FEEDBACK LINEARIZATION CONTROL}

Simulations results of the feedback linearized tank system as in (Figure.3) demonstrates both trackingand good regulation objectives are achieved, no excess is recorded in both transient and steady state which are well shown by the level error, moreover the chosen Lyapunov candidate function show that the global uniform exponential stability of the equilibrium point (guaranteed stability), and from the level error (Figure.4) we see that the system is also nearly robust to the rejected noise. 
Informatics Engineering, an International Journal (IEIJ), Vol.3, No.4, December 2015

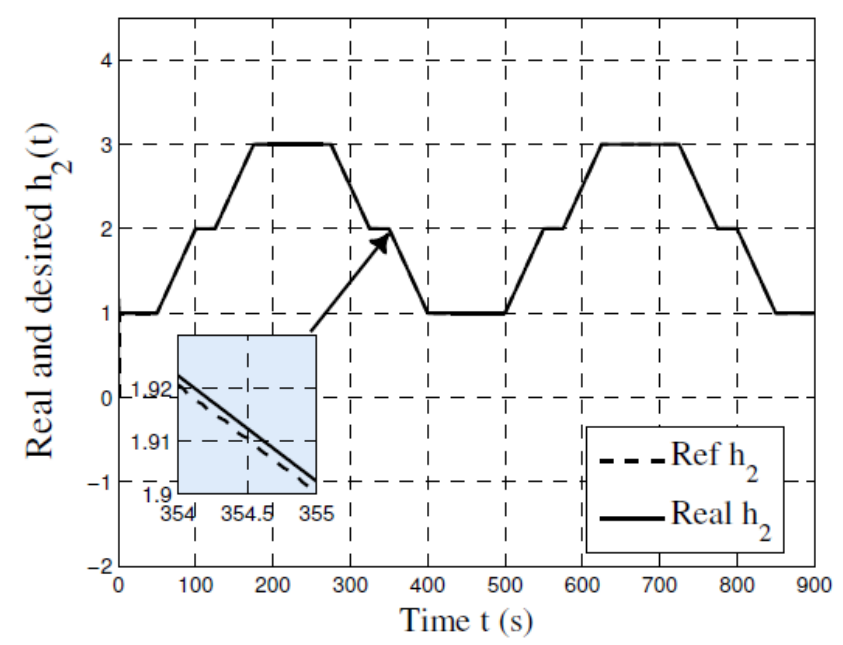

Figure 3: The level tracking for feedback linearization control

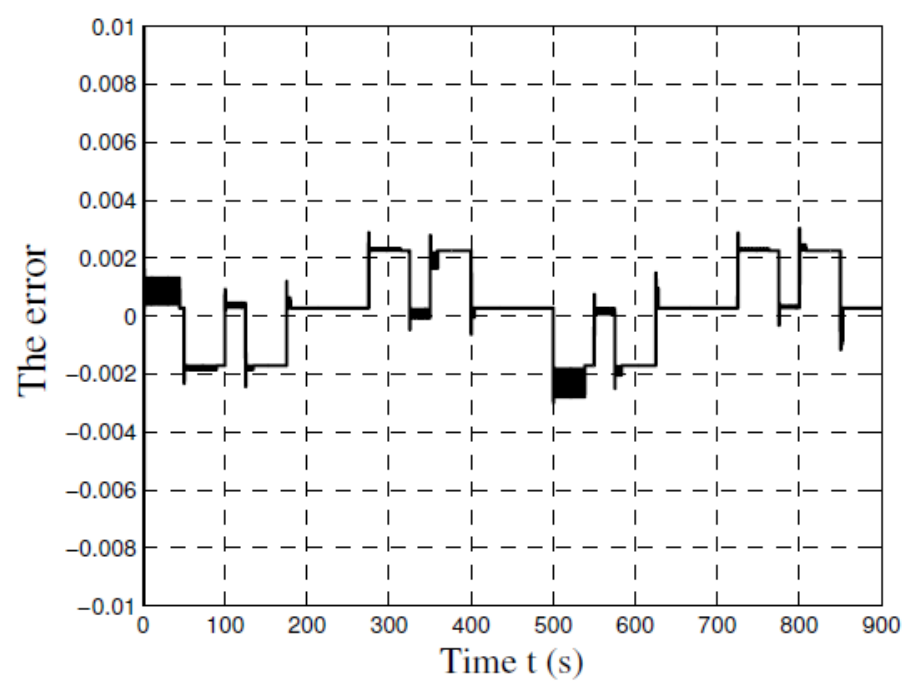

Figure 4: The level error for feedback linearization control 


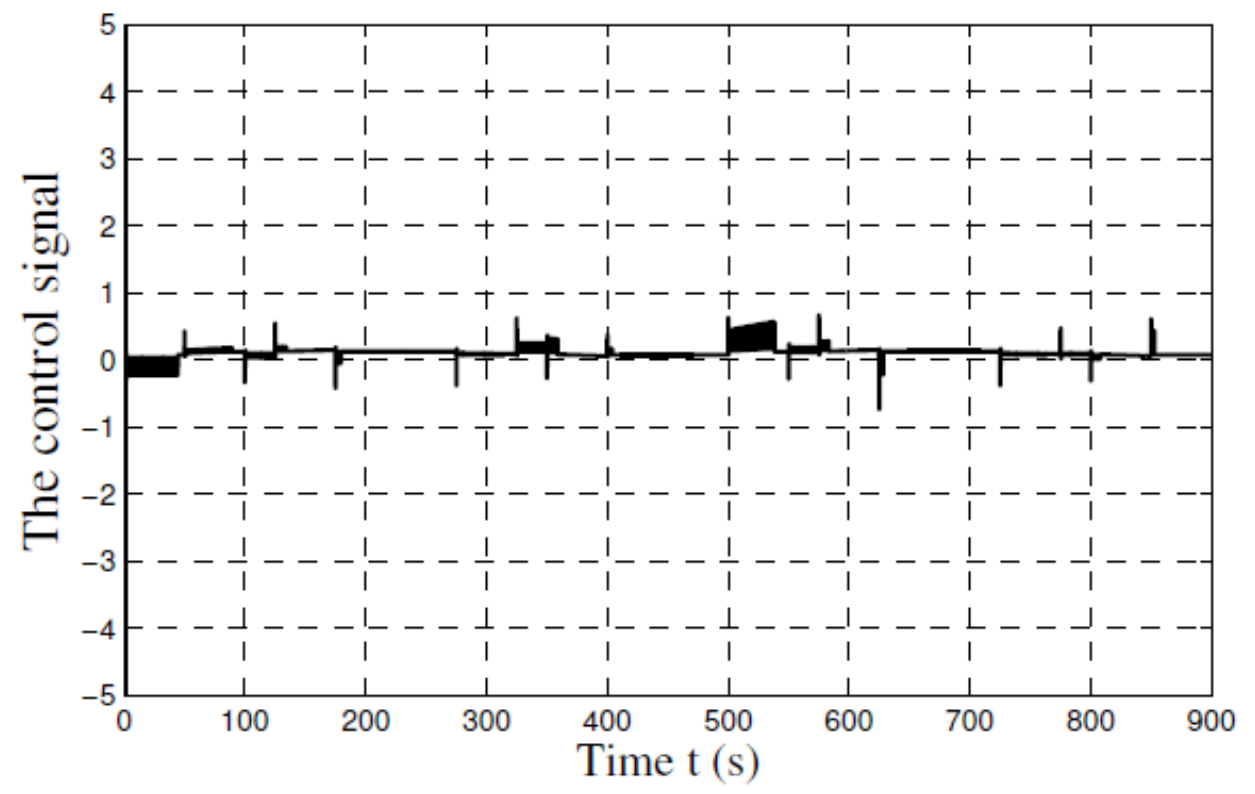

Figure 5: The control signal of feedback linearization control

\section{BACKSTEPPING APPROACH}

The backstepping was developed by Kanellakopoulos [12], and inspired by the work of Feurre\& Morse [13] on the one hand and Tisinias and Kokotovic\&Sussmann [14]. The basic idea of this method is to leave some states of the system act as virtual inputs. The backstepping uses a form of the system chain of integrators, after a coordinate transformation of a triangular system and based on the direct method of Lyapunov. The method is to split the system into a set of subsystem nested descending order. From there, it is possible to design systematically and recursively controllers and corresponding Lyapunov functions.

\subsection{THE SYNTHESIS OF BACKSTEPPING CONTROL}

The backstepping is a control law for nonlinear systems, based on Thelyapunov theory. It applies to systems of form triangular cascade. Each state of the system, control the previous state and called Virtual Control until the last state that is controlled by the actual control. The objective of this technique is to compute multistage a control law that provides the overall stability of the system [15],[16].

This control law takes the form of a recursive algorithm, the calculation steps are as follows:

The tracking errors are written in the form below 
Informatics Engineering, an International Journal (IEIJ), Vol.3, No.4, December 2015

$$
e_{x i}=\left\{\begin{array}{lc}
x_{i r}-x_{i} & i \text { impaire } \\
x_{i}-\dot{x}_{(i-1) r}-\alpha_{x(i-1)} e_{x(i-1)} & i \text { paire }
\end{array}\right.
$$

$$
\begin{array}{cl}
x_{i r} & : \text { The reference. } \\
x_{i} & : \text { State of system. } \\
\alpha_{x(i-1)} & : \text { A positive constant. } \\
e_{x(i-1)} & : \text { The error. }
\end{array}
$$

We consider the case of the the second order nonlinear systems of the form :

$$
\dot{x}=f(x)+g(x) u
$$

$x$ : State of system, $x \in \Re^{n}$.

$u$ : Control, $u \in \Re$.

Suppose that the system (23) after a transformation can be put under the chained form as follows:

$$
\begin{aligned}
& \dot{x}_{1}=f_{1}\left(x_{1}\right)+g_{1}\left(x_{1}\right) x_{2} \\
& \dot{x}_{2}=f_{2}\left(x_{2}\right)+g_{2}\left(x_{2}\right) u
\end{aligned}
$$

We desired to follow the output $\mathrm{y}=\mathrm{x}_{1}$ the trajectory $\mathrm{y}_{\mathrm{r}}$. This trajectory is assumed known and uniformly bounded and since the system is a second order the design is done in two steps:

\section{First iteration}

First consider the equation (24), where $\mathrm{x}_{2}$ the state variable is treated as a virtual control, and we Defined like the first desired value.

$$
x_{1 \mathrm{~d}}=y_{r}
$$

The first error variable is defined by:

$$
\begin{aligned}
& e_{1}=x_{1}-x_{1 \mathrm{~d}} \\
& \dot{e}_{1}=\dot{x}_{1}-\dot{x}_{1 \mathrm{~d}}
\end{aligned}
$$

With equation (28), the equation system (22) is written:

$$
\dot{e}_{1}=f_{1}\left(x_{1}\right)+g_{1}\left(x_{1}\right) x_{2}-\dot{x}_{1 \mathrm{~d}}
$$

For such a system, it was shown that the candidate function is a good choice of the Lyapunov function: 


$$
V_{1}\left(e_{1}\right)=\frac{1}{2} e_{1}^{2}
$$

Its derivative is given by:

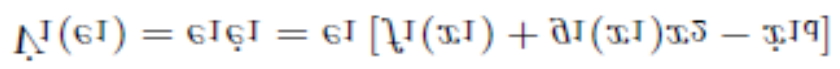

A judicious choice of $\mathrm{x}_{2}$ would render negative $\mathrm{V}^{\cdot}{ }_{1}\left(\mathrm{e}_{1}\right)$ and ensures the stability of the origin of the subsystem described by equation (24).

Take for value $x_{2}$, the function $t_{1}$, suchas:

$$
f_{1}\left(x_{1}\right)+g_{1}\left(x_{1}\right) t_{1}-\dot{x}_{1 \mathrm{~d}}=-k_{1} e_{1}
$$

Where $\mathrm{k} 1>0$ is a design parameter.

Equation (32) implies that the condition:

$$
\ddot{V}_{1}\left(e_{1}\right)=-k_{1} e_{1}^{2} \leqslant 0
$$

This implies that the error given by equation (27) tends to zero which ensures the convergence of thesubsystem (24) and its asymptotic stability.

From Equation (32) we obtained:

$$
x_{2 \mathrm{~d}}=t_{1}=\frac{1}{g_{1}\left(x_{1}\right)}\left[-k_{1} e_{1}+\dot{x}_{1 \mathrm{~d}}-f_{1}\left(x_{1}\right)\right]
$$

\section{Second iteration}

We consider the subsystem equation (25) and the new error variable is defined:

$$
e_{2}=x_{2}-t_{1}
$$

Thisequationrepresentsthegapbetweenthestatevariable $x_{2}$ anditsdesiredvalue $t_{1}$, theerror $e_{2}$ is notinstantlynull.Theobjectiveofthisstepisthentoforceittocancel.

Theequationsofthesystemtobecontrolledinspace $\left(e_{1}, e_{2}\right)$, arewritten:

$$
\begin{gathered}
\dot{e}_{1}=f_{1}\left(x_{1}\right)+g_{1}\left(x_{1}\right)\left(e_{2}+t_{1}\right)-\dot{x}_{1 \mathrm{~d}} \\
\dot{e}_{2}=f_{2}\left(x_{2}\right)+g_{2}\left(x_{2}\right) u-\dot{t}_{1}
\end{gathered}
$$


Lyapunov function is chosen as:

$$
V_{2}\left(e_{1}, e_{2}\right)=V_{1}\left(e_{1}\right)+\frac{1}{2} e_{2}^{2}
$$

its derivative

$$
\begin{gathered}
\dot{V}_{2}\left(e_{1}, e_{2}\right)=\dot{V}_{1}+e_{2} \dot{e}_{2}=e_{1} \dot{e}_{1}+e_{2} \dot{e}_{2} \\
\dot{V}_{2}\left(e_{1}, e_{2}\right)=e_{1}\left[f_{1}\left(x_{1}\right)+g_{1}\left(x_{1}\right)\left(e_{2}+t_{1}\right)-\dot{x}_{1 \mathrm{~d}}\right]+ \\
e_{2}\left[f_{2}\left(x_{2}\right)+g_{2}\left(x_{2}\right) u-\dot{t}_{1}\right] \\
\dot{V}_{2}\left(e_{1}, e_{2}\right)=e_{1}\left[f_{1}\left(x_{1}\right)+g_{1}\left(x_{1}\right) e_{2}+g_{1}\left(x_{1}\right) t_{1}-\dot{x}_{1 \mathrm{~d}}\right] \\
+e_{2}\left[f_{2}\left(x_{2}\right)+g_{2}\left(x_{2}\right) u-\dot{t}_{1}\right] \\
\dot{V}_{2}\left(e_{1}, e_{2}\right)=- \\
K_{1} e_{1}^{2}+e_{2}\left[f_{2}\left(x_{2}\right)+g_{2}\left(x_{2}\right) u-\dot{t}_{1}+e_{1}\right]
\end{gathered}
$$

Toensurestabilityitisnecessarythattheequationisequalto $-k 2 e 2$ with $K 2>0$

$$
f_{2}\left(x_{2}\right)+g_{2}\left(x_{2}\right) u+g_{1}\left(x_{1}\right) e_{1}-\dot{t}_{1}+e_{1}=-K_{2} e_{2}
$$

The control is:

$$
u=\frac{1}{g_{2}\left(x_{2}\right)}\left[-K_{2} e_{2}+\dot{t}_{1}-e_{1}-g_{1}\left(x_{1}\right) e_{1}-f_{2}\left(x_{2}\right)\right]
$$

With this choice of control u we obtained:

$$
\dot{V}_{2}\left(e_{1}, e_{2}\right)=-K_{1} e_{1}^{2}-K_{2} e_{2}^{2}
$$

So finally, we guarantee the asymptotic stability of the system. 
Informatics Engineering, an International Journal (IEIJ), Vol.3, No.4, December 2015

Let,

$$
\left\{\begin{array} { l } 
{ z _ { 1 } = x _ { 1 } } \\
{ z _ { 2 } = x _ { 2 } - x _ { 1 } }
\end{array} \Leftrightarrow \left\{\begin{array}{l}
\dot{z}_{1}=B_{1} \sqrt{z_{2}}-B_{2} \sqrt{z_{1}} \\
\dot{z}_{2}=B_{2} \sqrt{z_{1}}-2 B_{1} \sqrt{z_{2}}+k_{a} u
\end{array}\right.\right.
$$

And, another transformation

$$
\begin{gathered}
L=T(z) \\
\left\{\begin{array} { l } 
{ L _ { 1 } = z _ { 1 } } \\
{ L _ { 2 } = \dot { z } _ { 1 } = \dot { L } _ { 1 } }
\end{array} \Leftrightarrow \left\{\begin{array}{l}
L_{1}=z_{1} \\
L_{2}=B_{1} \sqrt{z_{2}}-B_{2} \sqrt{z_{1}}
\end{array}\right.\right. \\
\left\{\begin{array}{l}
\dot{L}_{1}=L_{2} \\
\dot{L}_{2}=\frac{B_{1} \dot{z}_{2}}{2 \sqrt{z_{2}}}-\frac{B_{2} \dot{z}_{1}}{2 \sqrt{z_{1}}}
\end{array}\right.
\end{gathered}
$$

$$
\left\{\begin{array}{l}
\dot{L}_{1}=L_{2} \\
\dot{L}_{2}=\frac{B_{1} B_{2}}{2}\left[\sqrt{\frac{z_{1}}{z_{2}}}-\sqrt{\frac{z_{2}}{z_{1}}}\right]-B_{1}{ }^{2}+\frac{B_{2}{ }^{2}}{2}+\frac{B_{1} k_{a}}{2 \sqrt{z_{2}}} u
\end{array}\right.
$$

So,

$$
\left\{\begin{array}{l}
\dot{L}_{1}=L_{2} \\
\dot{L}_{2}=h+\psi u \\
y=k_{s} L_{1}
\end{array}\right.
$$

Where

$$
h=\frac{B_{1} B_{2}}{2}\left[\sqrt{\frac{z_{1}}{z_{2}}}-\sqrt{\frac{z_{2}}{z_{1}}}\right]-B_{1}^{2}+\frac{B_{2}^{2}}{2}, \quad \psi=\frac{B_{1} k_{a}}{2 \sqrt{z_{2}}}
$$

We choose the error and the lyapunov candidate function as follow :

$$
\left\{\begin{array} { l } 
{ e _ { 1 } = k _ { s } \mathrm { L } _ { 1 } - y _ { d } } \\
{ e _ { 2 } = L _ { 2 } - t _ { 1 } , / t _ { 1 } = K _ { 1 } e _ { 1 } }
\end{array} \left\{\begin{array}{l}
V_{1}\left(e_{1}\right)=\frac{1}{2} e_{1}^{2} \\
V_{2}\left(e_{2}\right)=\frac{1}{2} e_{1}^{2}+\frac{1}{2} e_{2}^{2}
\end{array}\right.\right.
$$

The control law is:

$$
u=\frac{\dot{t}_{1}-h+K_{2} e_{2}}{\psi}
$$

Shows the implementation of this Backstepping in (Figure.6) 


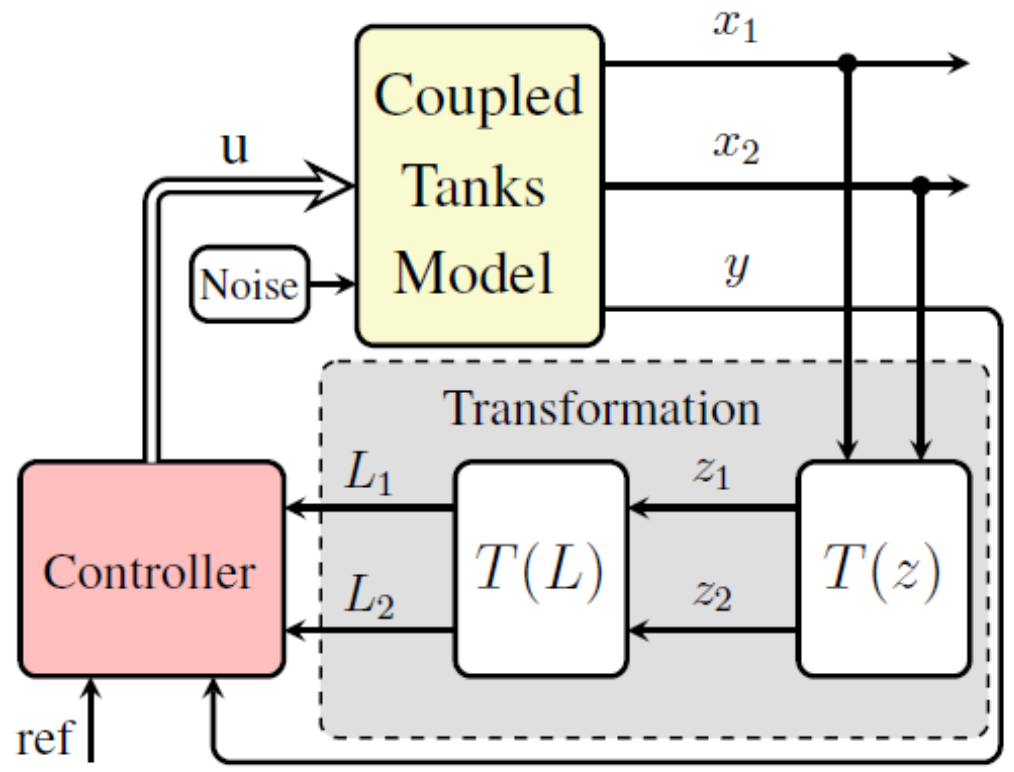

Figure 6: Schematic block diagram of Backstepping control

\subsection{SIMULATION RESULTS OF BACKSTEPPING CONTROL}

After seeing these results, we can conclude that :

The Backstepping is a control technque that ensures the stability within the Lyapunov sense. Thetechnique provides a flexible handling, we see the level coincides with its reference ( best tracking), (Figure.7) no overshooting, no statistical error, no excess is recorded in both transient and at steady state, which are well shown by the error (Figure.8).

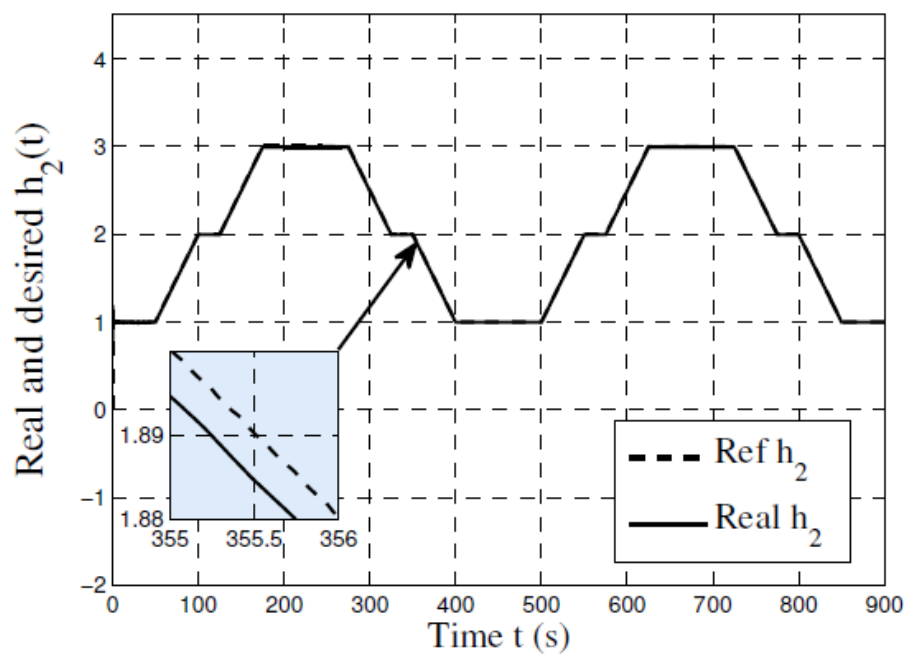

Figure 7: The level tracking for backsteppingn control 
Informatics Engineering, an International Journal (IEIJ), Vol.3, No.4, December 2015

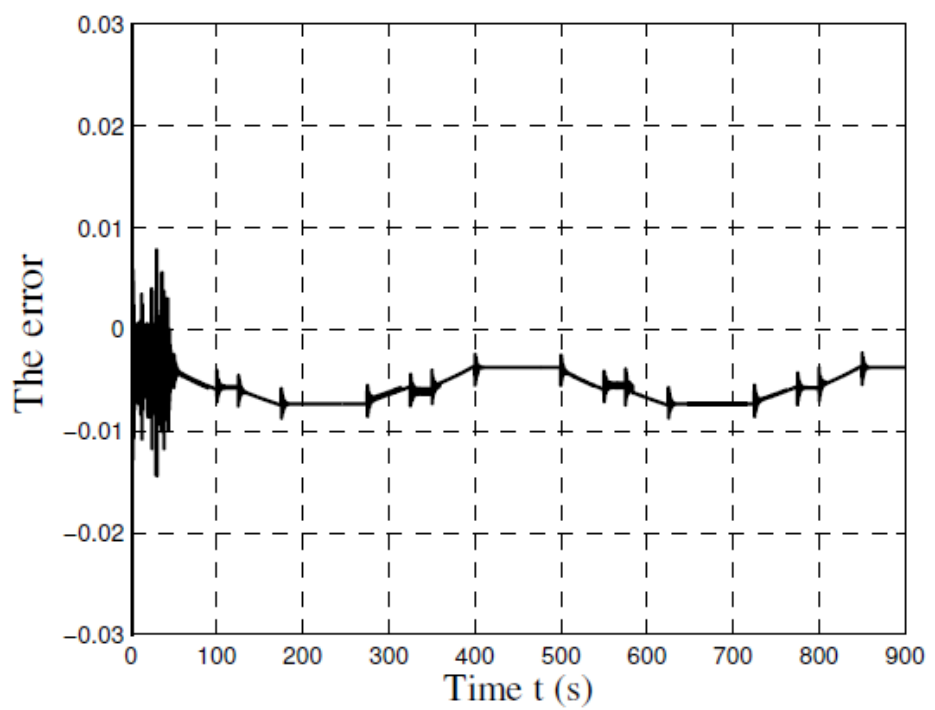

Figure 8: The level error for backstepping control

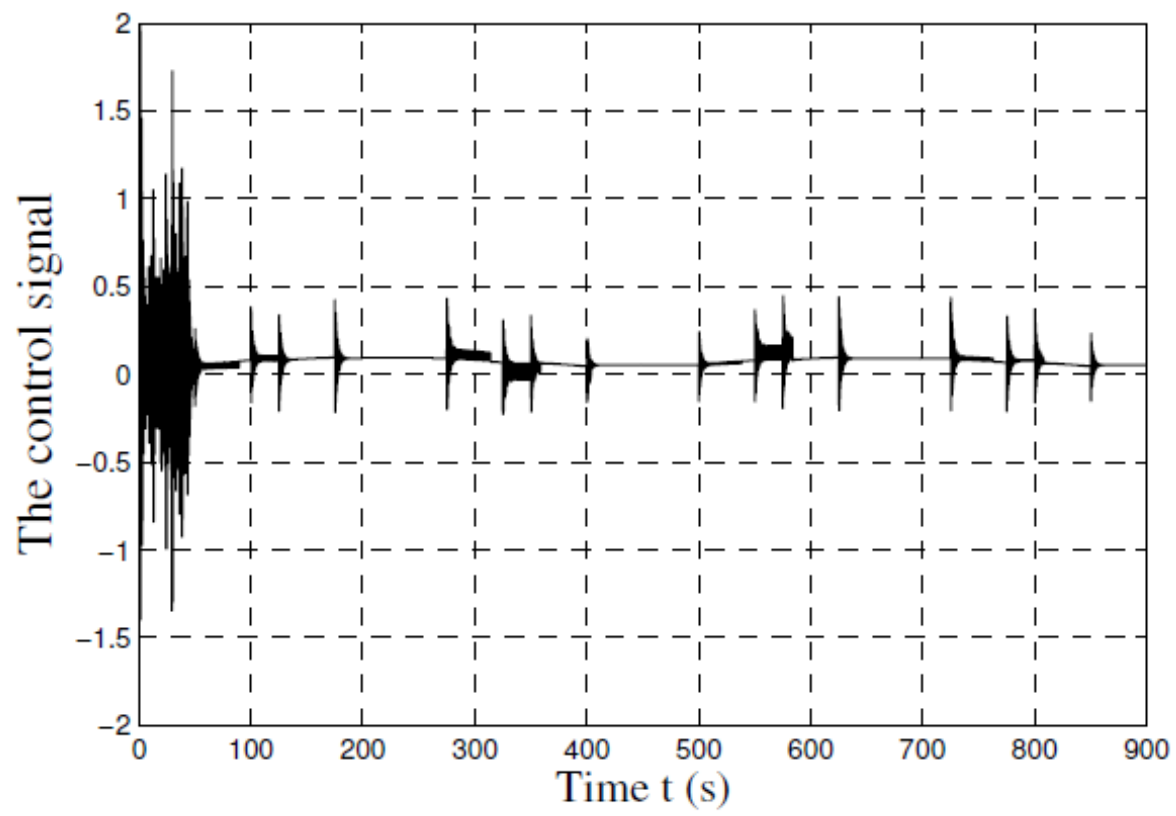

Figure 9: The control signal of backstepping control 


\section{CONCLUSION}

In this paper two types of nonlinear control schemes has been presented in detailed manner and applied to coupled tanks system. Concerning the feedback linearization controller, it can be said from the results has been done satisfactory and the simulations demonstrate a good performance of regulation and tracking. The total coupled tanks system is globally asymptotic stable and maintained nearly robust against perturbations. For the second controller approximately the same obtained results only the difference in the robustness which is preserved by the later scheme with less restrictive conditions.

The choice of the coefficients (controller gains) can be very tricky and difficult in the two approaches(FLC,BC) although these are selected positive but may induce instability problem, even in the chosen that ensure system stability, they can make signal very energetic order .

Finally in order of the work completion there are several points in this paper which may be naturalpoints of departure for further studies, the investigation of other methods rather than the used ones provides future works. If we consider the parametric variation in certain interval the hyper-stability adaptive control will be more efficient from the identifiability and the stability point of view.

Table 1: Parameters and variables of the coupled tanks, and the controllers (FLC,BC)

\begin{tabular}{|clc|}
\hline Notation & Parameter & Value \\
\hline$c$ & Tank sectional area & $9350 \times 10^{-6} \mathrm{~m}^{2}$ \\
$s_{1}, s_{2}$ & Channel sectional area & $78.5 \times 10^{-6} \mathrm{~m}^{2}$ \\
$a_{i}$ & Discharge input coefficient & $\simeq 1$ \\
$a_{s}$ & Discharge output coefficient & $\simeq 1$ \\
$k_{p}$ & Pump gain & $7.5 \mathrm{~m}^{3} / \mathrm{s} . \mathrm{v}$ \\
$k_{s}$ & Sensor gain & $40 \mathrm{v} / \mathrm{m}$ \\
$g$ & Gravity constant & $9.8 \mathrm{~m} / \mathrm{s}^{2}$ \\
$h_{\max }$ & Maximal liquid level & $0.25 \mathrm{~m}$ \\
$u_{\max }$ & Maximal entry voltage & $10 \mathrm{v}$ \\
$K_{p}$ & Position constant gain & 100 \\
$K_{v}$ & Speed constant gain & 400 \\
$K_{1}$ & Backstepping gain & 8.2 \\
$K_{2}$ & Backstepping gain & 0.2 \\
\hline
\end{tabular}


Informatics Engineering, an International Journal (IEIJ), Vol.3, No.4, December 2015

\section{REFERENCES}

[1] B.Nail, B.Bekhiti, K.Bdirina, A.Kouzou, and A.Hafaifa, Sliding Mode Control And Optimal GPC Algorithm for Coupled Tanks, 3rd International Conference on Control, Engineering \& Information Technology CEIT'2015 Tlemcen, Algeria, 25-27 May, 2015.

[2 ]Jiffy Anna John, N. E. Jaffar and Riya Mary Francis, Modelling and Control of Coupled Tank Liquid Level System using Backstepping Method, International Journal of Engineering Research \& Technology (IJERT), Vol. 4 Issue 06, pp. 667-671, June-2015.

[3] HéctorBastida, Pedro Ponce, Ricardo Ramìrez and Arturo Molina, Model and Control for Coupled Tanks using Labview, 2013 International Conference on Mechatronics, Electronics and Automotive Engineering, 127-133 IEEE 2013.

[4] AhceneBoubakir, Fares Boudjema and Salim Labiod, A Neuro-fuzzy-sliding Mode Controller Using Nonlinear Sliding Surface Applied to the Coupled Tanks System, International Journal of Automation and Computing, Vol 6 issue 1, pp. 72-80. February 2009.

[5] N.K.Poulsen, B.Kouvaritakis and M.Cannon, Nonlinear constrained predictive control applied to a coupled-tanks apparatus, IEE Proceedings - Control Theory and Applications Vol 148 issue 1, pp. 17-24. 2001.

[6] Isidori A, W. Kang, H- control via measurement feedback in nonlinear systems,IEEE Trans. On Automatic control, Vol. 40, pp. 466 - 472, 1995.

[7] Isidori A. and A. Astolfi, disturbance attenuation and $\mathrm{H}$ - control via measurement feedback in nonlinear systems, IEEE Trans Automatic control, AC - 37, pp. 1283-1293,1992.

[8] M. KrstiC, I. Kanellakopoulos, and P. Kokotovic.Nonlinear and Adaptive Control Design.WileyInterscience Publication, 1995.

[9] H. K. Khalil, Nonlinear Systems, 3rd ed. Upper Saddle River, NJ: Prentice-Hall, 2002.

[10] JJE. Slotine, W. Li, Applied Nonlinear Control, Englewood Cliffs, NJ: Prentice Hall, 1991.

[11] Vidyasagar M., Nonlinear Systems Analysis - Second edition, New Jersey: prentice Hall Inter, 1992.

[12] I. Kanellakopoulos, systematic design of adaptive controllers for feedback linearisable Systems, IEEE Transaction on automatic control vol 36(11), pp 1241-1253, July 2000.

[13] A. Feurrel\& A.S. Morse, Adaptive control of single-input, single-output linear systems, IEEE. Transaction on automatic control vol 23 (4), pp 557-569, July 2000.

[14] P.V, Kokotovic and H.J, Sussman, A positive real condition for global stabilization of nonlinear systems, systems \& control letters vol.13, pp 125-133, July 2000.

[15] Alexandra Bento Moutinho, Modeling and Nonlinear Control for Airship Autonomous Flight, university LISBOA December 2007.

[16] Guoqiang Wu, BaoJun Lin, Shancong Zhang, Fault-Tolerant Backstepping Attitude Control for Autonomous Airship with Sensor Failure, Procedia Engineering, vol 29, p 2022 - 2027, 2012.

[17] W. Bolek, J.Sasiadek, Singularity of backstepping control for non-linear systems, American Control Conference, Vol 4, pp2689-2694, 8-10 May 2002.

[18 ]ChangyunWen, Jing Zhou. Decentralized adaptive backsteppingstabilization of interconnected systems with dynamic input and output interactions, Automatica, Vol.45, 55-67, 2009.

[19] A. Benaskeur, L.N. Paquin, and A. Desbiens.Toward industrial control applications of the backstepping.Process Control and Instrumentation, Glasgow, Scotland, pages 62-67,2000.

[20 ]R. Sepulchre, M. Jankovic, and P. Kokotovic. Constructive Nonlinear Control.Springer VerlagLondon, 1997.

[21] Kokotovic P.V., Sussmann H.J, A Positive Real Condition for Global Stabilization of Nonlinear Systems, Systems and Control Letters, vol 13, pp 125-133, 1989. 